OPEN ACCESS

Edited by:

Christine Marosi,

Medical University of Vienna, Austria

Reviewed by:

Teresa Somma,

Federico II University Hospital, Italy Giovanni Raffa,

University of Messina, Italy

*Correspondence:

Giuseppe Broggi

giuseppe.broggi@gmail.com

Specialty section:

This article was submitted to Neuro-Oncology and Neurosurgical

Oncology,

a section of the journal

Frontiers in Neurology

Received: 26 November 2021

Accepted: 11 January 2022

Published: 09 February 2022

Citation:

Broggi G, Piombino E, Altieri R, Romano C, Certo F, Barbagallo GMV, Vigneri P, Condorelli D, Colarossi L,

Colarossi C, Magro G and Tirrò $E$ (2022) Glioblastoma, IDH-Wild Type With FGFR3-TACC3 Fusion: When Morphology May Reliably Predict the Molecular Profile of a Tumor. A Case Report and Literature Review.

Front. Neurol. 13:823015 doi: 10.3389/fneur.2022.823015

\section{Glioblastoma, IDH-Wild Type With FGFR3-TACC3 Fusion: When Morphology May Reliably Predict the Molecular Profile of a Tumor. A Case Report and Literature Review}

\author{
Giuseppe Broggi ${ }^{1 *}$, Eliana Piombino ${ }^{2}$, Roberto Altieri ${ }^{3}$, Chiara Romano ${ }^{4,5}$, \\ Francesco Certo ${ }^{3}$, Giuseppe Maria Vincenzo Barbagallo ${ }^{3}$, Paolo Vigneri ${ }^{4,5}$, \\ Dario Condorelli ${ }^{6}$, Lorenzo Colarossi ${ }^{2}$, Cristina Colarossi ${ }^{2}$, Gaetano Magro ${ }^{1}$ and \\ Elena Tirrò ${ }^{5,7}$
}

${ }^{1}$ Department of Medical and Surgical Sciences and Advanced Technologies "G.F. Ingrassia", Anatomic Pathology, University of Catania, Catania, Italy, ${ }^{2}$ Pathology Unit, Department of Experimental Oncology, Mediterranean Institute of Oncology, Catania, Italy, ${ }^{3}$ Department of Medical and Surgical Sciences and Advanced Technologies "G.F. Ingrassia", Neurological Surgery, Policlinico "G. Rodolico-San Marco" University Hospital, University of Catania, Catania, Italy, ${ }^{4}$ Department of Clinical and Experimental Medicine, University of Catania, Catania, Italy, ${ }^{5}$ Center of Experimental Oncology and Hematology, A.O.U. Policlinico "G. Rodolico-San Marco", Catania, Italy, ${ }^{6}$ Department of Medical and Surgical Sciences and Advanced Technologies "GF Ingrassia", University of Catania, Catania, Italy, ${ }^{7}$ Department of Surgical, Oncological and Stomatological Sciences, University of Palermo, Palermo, Italy

It has been reported that in-frame FGFR3-TACC3 fusions confer to glioblastomas, $I D H$-wild type (GBMs, IDHwt) some unusual morphologic features, including monomorphous rounded cells with ovoid nuclei, nuclear palisading, endocrinoid network of "chicken-wire" vessels, microcalcifications and desmoplastic stroma, whose observation may predict the molecular profile of the tumor. We herein present a case of recurrent GBMs, IDHwt, exhibiting some of the above-mentioned morphological features and a molecularly-proven FGFR3-TACC3 fusion. A 56-year-old man presented to our hospital for a recurrent GBM, IDHwt, surgically treated at another center. Histologically, the tumor, in addition to the conventional GBM morphology, exhibited the following peculiar morphologic features: (1) monomorphous neoplastic cells with rounded nuclei and scant pale cytoplasm; (2) thin capillary-like vessels with "chicken-wire" pattern; (3) nuclear palisading; (4) formation of vague perivascular pseudorosettes; (5) spindled tumor cells embedded in a loose, myxoid background. Based on this unusual morphology, molecular analyses were performed and an FGFR3 exon17-TACC3 exon 10 fusion was found. The present case contributes to widening the morphologic spectrum of FGFR3-TACC3-fused GBM, IDHwt and emphasizes that pathologists, in the presence of a GBM, IDHwt with unconventional morphology, should promptly search for this fusion gene.

Keywords: FGFR3-TACC3 fusion, glioblastoma, unusual morphological features, molecular biology, diagnosis, IDH-wildtype, high-grade glioma 


\section{INTRODUCTION}

Adult glioblastomas, $I D H$-wild type comprise a molecularly and histopathologically heterogeneous spectrum of neoplasms, characterized by poor prognosis and frequent resistance to the conventional radio-chemotherapy treatments (1-3). According to the cIMPACT-NOW criteria (4), the molecular diagnosis of glioblastomas, $I D H$-wild type (GBMs, $I D H w t)$ is essentially based on the presence of at least one of the following alterations in the context of an adult diffuse astrocytic neoplasm, $I D H$-wt: i) combined $7 p$ gain and $10 q$ loss, ii) epidermal growth factor receptor (EGFR) amplification, and iii) telomerase reverse transcriptase (TERT) promoter mutation $(5,6)$. GBM, $I D H$ wt also shows a wide morphological spectrum, and some histopathologic variants exhibit additional molecular alterations with potential therapeutic implications (7). Genomic profiling studies revealed that GBMs show an extensive molecular heterogeneity and about $30-50 \%$ of malignant gliomas harbor targetable gene fusion mainly involving EGFR, neurotrophic tyrosine receptor kinase (NTRK), and fibroblast growth factor receptor (FGFR) genes (8). In the past, fibroblast growth factor receptor 3 (FGFR3)-transforming acidic coiled-coil 3 (TACC3) gene fusion was identified as a rare molecular feature in grade 1 to 4 adult diffuse gliomas lacking $I D H 1 / 2$ mutations but always carrying TERT promoter mutations or CDKN2A loss in about $75 \%$ of cases $(9,10)$. The FGFR3-TACC3 gene fusion acts as an oncogene, encoding a protein, located on mitotic spindle poles, with constitutive kinase function, that causes a loss of the normal chromosomal segregation and stimulates aneuploidy (11). The identification of the oncogenic FGFR3-TACC3 fusion highlighted the possibility of identifying a subset of diffuse glioma patients potentially responsive to targeted therapy with FGFR kinase inhibitors $(12,13)$. In the last few years, Bielle et al. have described a series of 30 adult high-grade diffuse gliomas, harboring an in-frame FGFR3-TACC3 fusion and exhibiting the conventional molecular alterations of GBMs, $I D H w t$, but peculiar histopathologic features; interestingly, the following unusual morphological features were found: "monomorphous ovoid nuclei, nuclear palisading, and thin parallel cytoplasmic processes, an endocrinoid network of thin capillaries) associated with frequent microcalcifications and desmoplasia" (14). Since then, additional cases with the co-occurrence of FGFR3-TACC3 fusion and the above-mentioned histopathologic features have been reported in the literature (15), raising the question of whether this unusual morphology may predict the presence of this equally rare molecular finding.

We herein report a case of a 56-year-old male patient affected by a recurrent GBM, IDHwt, showing both an unconventional morphology and a molecularly-proven FGFR3-TACC3 gene fusion. A critical review of the literature that emphasizes the potential association between morphology and molecular status of this GBM subtype is also included.

\section{CASE PRESENTATION}

A 56-year-old man was admitted to our hospital on March 2021 for the recurrence of a GBM, IDHwt, which had been surgically treated with a subtotal resection at another center in October 2017. After Stupp regimen and some months of wellness, he developed aphasia and confusion. Brain MRI showed a left parieto-occipital mass with infiltration of the splenium of the corpus callosum (Figure 1A) and a gross total resection with a good clinical result was surgically achieved (Figure 1B).

Histologically, the tumor was composed of spindled to rounded astrocytic cells that showed an infiltrating growth pattern and high-grade features, such as hypercellularity, high mitotic index (nine mitoses per 10 high-power fields), foci of microvascular proliferation, and pseudopalisading necrosis (Figure 2A). Interestingly, the tumor also exhibited some unusual morphological features (Figures 2B-D): i) presence of monomorphous ovoid cells with rounded nuclei and sometimes scant pale cytoplasm; ii) numerous thin capillary-like vessels with "chicken-wire" pattern, arranged in an endocrinoid pattern; iii) nuclear palisading; iv) focal perivascular arrangement of neoplastic cells, resulting in the formation of vague perivascular pseudorosettes; v) spindled neoplastic cells embedded in a loose, myxoid background, producing a "tissue culture-like" appearance. Neither microcalcifications, desmoplastic stroma, nor histologic signs of previous treatments were seen. The above-mentioned unusual morphological features were found both distant and in close proximity to tumor areas containing foci of necrosis and microvascular proliferation (Figure 2B). Neoplastic cells were diffusely stained with GFAP and OLIG2. No immunoexpression of IDH1 R132H, H3K27M, H3G34M, and CD34 was found. Nuclear expressions of ATRX and H3K27me3 were retained; $<10 \%$ of the neoplastic cells were stained with $\mathrm{p} 53$ and the Ki-67 proliferation rate was about $10 \%$. Based on both morphological and immunohistochemical features, a diagnosis of recurrent "WHO grade 4 glioblastomas, IDH-wild type" was rendered.

Subsequently, because of the unusual morphology encountered, next-generation sequencing (NGS) was chosen to identify further molecular alterations. NGS was performed using a custom panel for the identification of point mutations, INDEL and copy number variations (Glio-panel DNA), and a custom panel for the detection of gene fusions (Glio-panel RNA). The RNA sequencing of recurrent GBM revealed the presence of FGFR3 exon17-TACC3 exon 10 (Catalog of Somatic Mutations in Cancer mutation identifier COSF1434) fusion (Figure 3). Moreover, NGS sequencing identified the presence of the most common mutations associated with FGFR3-TACC3 fusion in GBM, IDHwt: the pathogenic deletion on the PTEN gene (p.Trp111Ter) and TERT c.C228T promoter mutation (16). Furthermore, chromosome 10q loss without chromosome seven gain was detected, while no EGFR, MDM2, and CDK4 amplification nor CDKN2A homozygous deletion were found in the analyzed sample.

\section{DISCUSSION}

FGFR3-TACC3 fusions are oncogenic drivers that were first reported in GBMs and bladder urothelial carcinomas (17); in more detail, this unusual fusion was first detected on a series of 


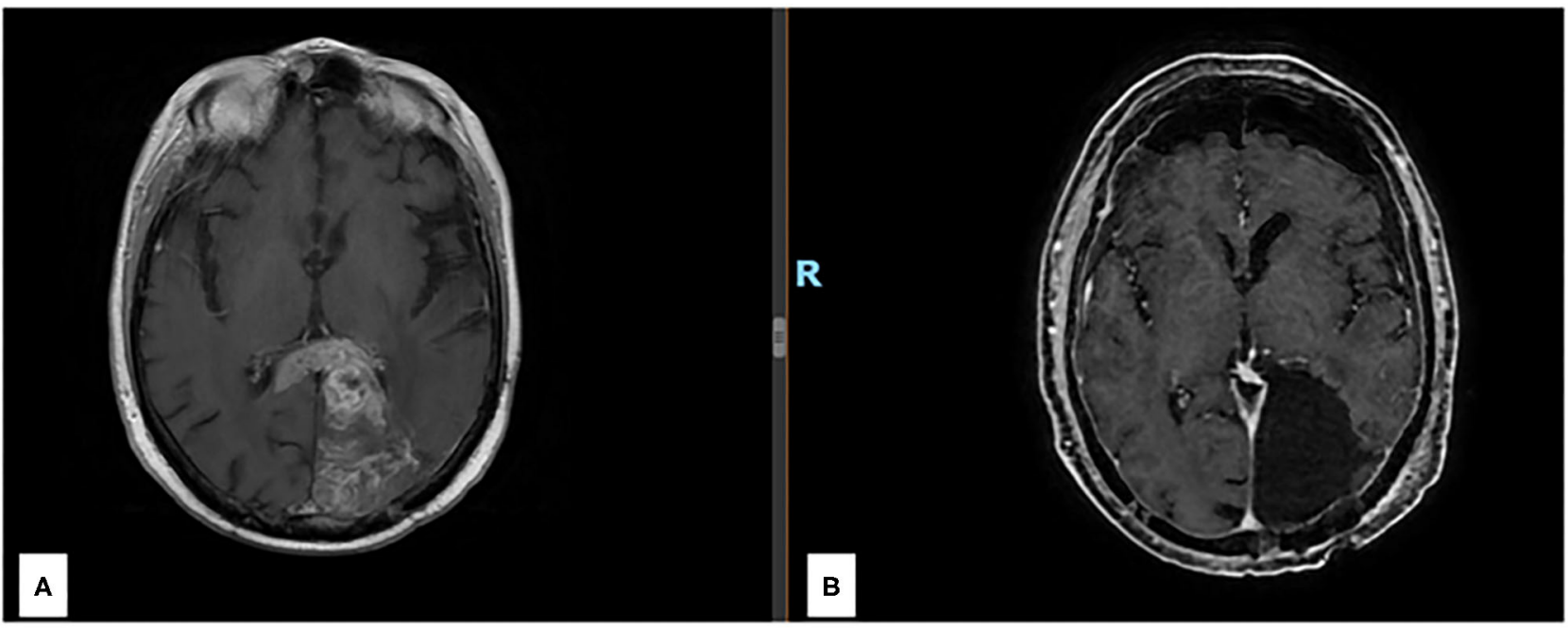

FIGURE 1 | (A) Preoperative axial section of a T1 w MRI after gadolinium injection revealing a left parieto-occipital recurrent lesion with infiltration of the splenium of the corpus callosum. (B) Postoperative axial section of a T1 w MRI after gadolinium injection revealing the complete resection of the enhancing nodule.

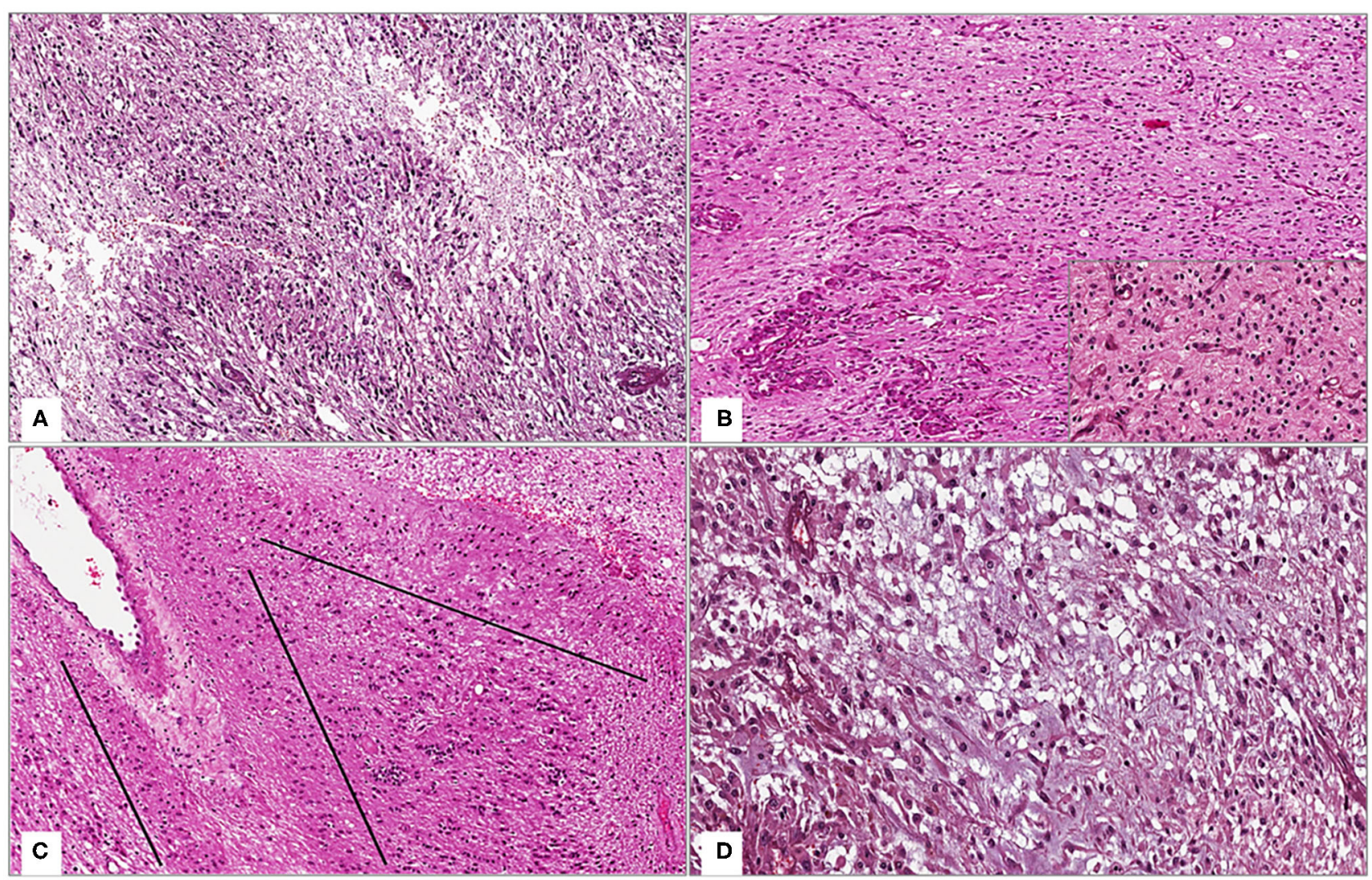

FIGURE 2 | (A) Low magnification showing the conventional morphology of WHO grade IV glioblastoma, IDH-wild type: a moderately cellular astrocytic tumor with foci of pseudopalisading necrosis (hematoxylin and eosin; original magnification 150x); (B) Tumor exhibits, as an unusual morphologic feature, more bland-looking areas composed of monomorphous round-shaped cells and thin capillary-like vessels with "chicken-wire" pattern, arranged in an endocrinoid pattern (insert); these features are also found close to foci of microvascular proliferation [hematoxylin and eosin; original magnifications 150x and 300x (insert)]; (C) Tumor areas with nuclear palisading (lines) are seen (hematoxylin and eosin; original magnification 150x); (D) Spindled neoplastic cells set in a loose, myxoid background, imparting to the tumor a focal "tissue culture-like" morphology (hematoxylin and eosin; original magnification 300x). 


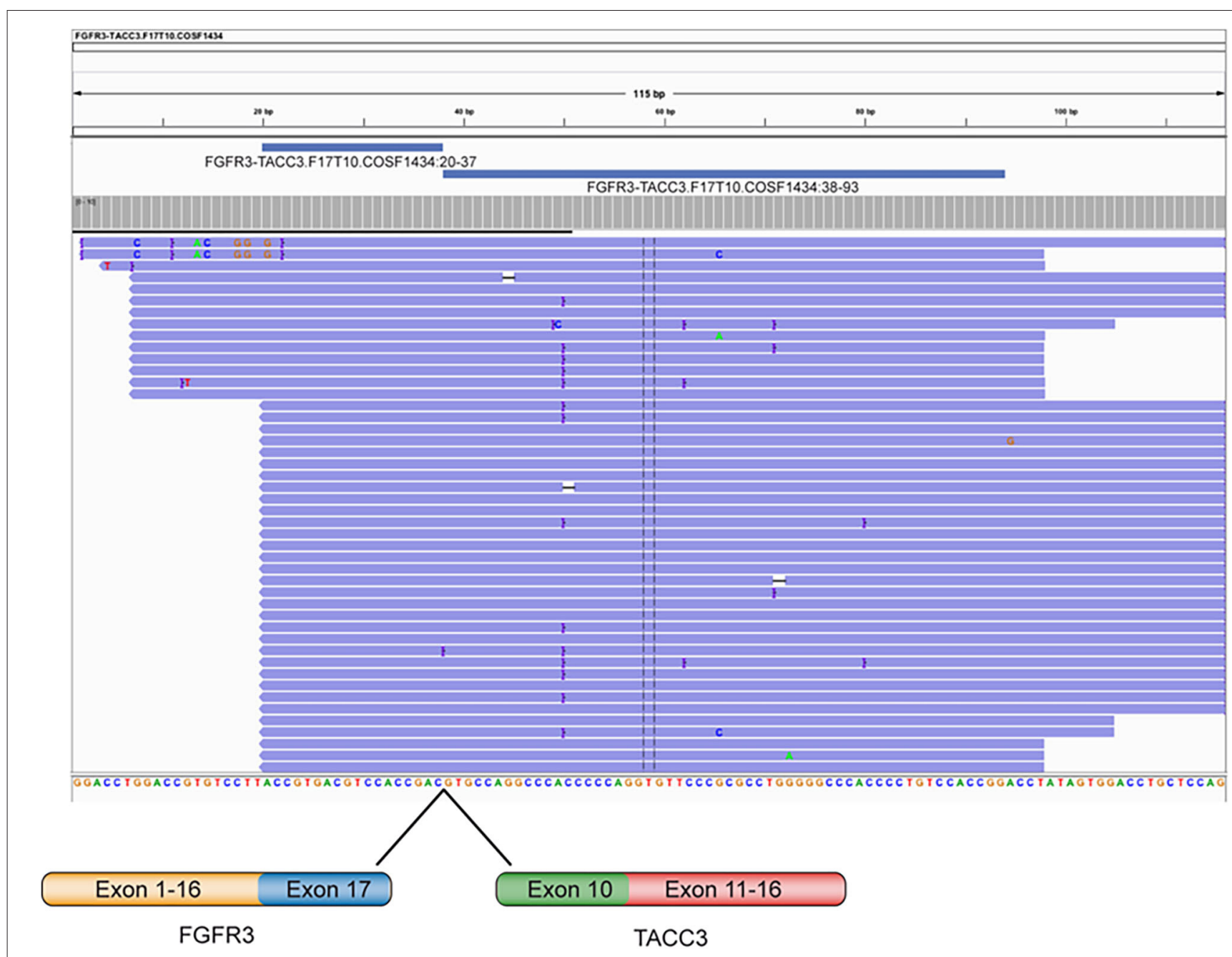

FIGURE 3 | Representation of the FGFR3-TACC3 fusion gene identified by Next Generation Sequencing in a GBM IDHwt patient. Visualization of RNA sequencing reads supports the fusion junction between FGFR3 exon 17 and TACC3 exon 10.

97 GBM cases, two of which harbored the FGFR3-TACC3 fusion (18). Subsequently, larger molecular studies on 584 GBMs and 211 lower-grade diffuse gliomas reported 17 GBMs and three lower-grade gliomas with FGFR3-TACC3 fusions (11). Based on other studies reported in the literature, it is estimated that only a small percentage of GBMs (1-8\%) harbor this gene fusion and the incidence decreases further if grade 2 and 3 diffuse gliomas are also considered (17). FGFR3-TACC3 fusions, although less frequently reported than FGFR2 and BRAF alterations, have also been identified in cases of "polymorphous low-grade neuroepithelial tumor of the young" (PLNTY) (19).

In 2017 Bielle et al. reported a series of 30 patients affected by FGFR3-TACC3-fused adult gliomas (age range: $42-87$ years), which exhibited some unusual morphological features, combined with microcalcifications and desmoplasia; their cohort included 25 cases of GBMs, IDHwt, one case of gliosarcoma, IDHwt, one case of GBM, not otherwise specified, two cases of diffuse astrocytomas "with molecular features of GBM" (7p gain, 10q loss, and TERT promoter mutation) and one case of histological grade 2 diffuse astrocytoma, IDHwt with no additional molecular analyses available (14). Furthermore, $73 \%$ of these cases showed some recurrent unusual morphological features, including monomorphous ovoid nuclei, endocrinoid network of capillary vessels, vague formation of perivascular pseudorosettes, nuclear palisading, microcalcifications, and desmoplastic stroma. The presence of this unusual morphology in GBM cases was restricted to areas that lacked necrosis and/or microvascular proliferation and extravascular immunohistochemical staining for CD34 was found in about $50 \%$ of cases. These tumors molecularly showed, in addition to the FGFR3-TACC3 fusion, the conventional GBM, IDHwt features (absence of IDH1/2, ATRX and TP53 mutations, 7p gain, 10q loss, and TERT promoter mutations), except for EGFR amplification $(0 / 29)$, combined with a higher incidence of CDKN2A homozygous deletions.

The study of Gilani et al. recently described the histopathologic features of six adult GBMs, IDHwt with FGFR3TACC3 fusion and lack of EGFR amplification, confirming the 
presence of the above-mentioned unusual morphologic features, variably combined, in five out of six cases. The remaining case, despite harboring the FGFR3-TACC3 fusion, exhibited a different morphology from that previously published, characterized by less "bland-looking" cellularity and more striking nuclear atypia (15). Despite being aware that the detection of monomorphous ovoid cells with endocrinoid network of vessels, microcalcifications, and desmoplasia on a high-grade glioma, IDHwt might justify the search for FGFR3-TACC3 fusions, the authors concluded that morphology alone could not predict the molecular status of these rare subsets of GBMs, as some FGFR3-TACC3-fused cases, that lacked these peculiar features, occurred, and, conversely, GBM cases, exhibiting this unusual morphology, lacked the FGFR3-TACC3 fusion.

The present paper reports an additional case of a recurrent GBM, IDHwt, and FGFR3-TACC3 fused with emphasis on the potential correlation between histopathology and molecular status. Histologically, our case showed tumor areas with conventional morphology of GBM, alternating with areas with some of the above-mentioned unusual morphological features. Compared to those cases reported in the literature, the present case showed, as an additional and previously unreported morphologic feature, a spindled neoplastic component, embedded in a loose, myxoid background, producing a "tissue culture-like" appearance. These particular histopathologic features were also found close to tumor areas with necrosis and foci of microvascular proliferation and led us to request a further molecular test for diagnostic confirmation and for the search of FGFR3-TACC3 fusion, whose presence has not only a speculative but also a practical function as it identifies a subset of patients with a slightly better prognosis than those affected by conventional GBM, IDHwt and who could benefit from a targeted therapy with FGFR kinase inhibitors. As some of these uncommon morphologic features are shared with other brain tumors, they often represent diagnostic challenges: i) oligodendrogliomas, $I D H$-mutant, and $1 \mathrm{p} / 19 \mathrm{q}$ codeleted often exhibit monomorphic rounded cells with pale cytoplasm and a "chicken-wire" vascular network; ii) ependymomas and angiocentric glioma characteristically show perivascular pseudorosettes; iii) glioneuronal tumors, in general, may exhibit extravascular positivity for CD34 and desmoplastic stroma (14). These histological findings in a diffuse glioma $I D H$-wildtype should prompt pathologists to consider FGFR3-TACC3 fusion and look for additional genetic alterations that are required for the diagnosis of GBM, IDHwt. The treatment for patients with GBM includes combined radio and chemotherapy (20). Temozolomide (TMZ) is the standard chemotherapeutic used alone or in association with a DNA alkylating agent: however, chemoresistance and not well-characterized mechanisms involved in the development of tumors are the most common cause of therapy failure (21-25). Furthermore, for recurrent gliomas, standard-of-care treatments are not well defined; treatment is usually selected based on prior therapy, age, Karnofsky Performance Scale (KPS), MGMT promoter methylation status, and patterns of disease progression (26). Bevacizumab, an anti-vascular endothelial growth factor (VEGF) monoclonal antibody that has been introduced in the
USA in 2009 as a treatment for recurrent high-grade gliomas, has become one of the first-choice therapies for recurrent GBMs, according to National Comprehensive Cancer Network (NCCN) guidelines. The combination of bevacizumab and chemotherapy represents an additional treatment option for these patients. However, when the standard therapeutic regimens lack efficacy, targeted therapies for patients with primary and recurrent GBMs are currently limited, and novel molecular biomarkers are needed to improve the development of personalized treatments.

$\mathrm{Xu}$ et al. reported that potentially targetable molecular alterations, mainly involving NTRK, EGFR, and FGFR genes, occurred in about 30 to $50 \%$ of GBMs (8). In more detail, while NTRK rearrangements are very rare, being found in $<2 \%$ of GBM cases and consisting of fusions between NTRK1 and other genes, such as NFASC, BCAN, CHTOP, and ARHGEF2, $E G F R$ in-frame fusions are much more frequent (EGFR-SEPT14 and EGFR-PSPH fusion genes were observed approximately in 4 and $2 \%$ of cases) and frequently lead to EGFR overexpression in GBM (8); however, all clinical trials with EGFR inhibitors did not demonstrate longer survival times in GBM patients so far, probably due to the inclusion of poorly homogeneous patient populations. Finally, these authors reported that about 1 to $8 \%$ of GBMs harbored potentially druggable FGFRTACC rearrangements, being FGFR3-TACC3 the gene fusion most frequently encountered in $5 \%$ of cases, followed by FGFR1-TACC1 (8). Nowadays, tyrosine kinase fusion genes are an important class of oncogenes associated with different hematological and solid tumors (27), thus targeting gene fusion has been a promising therapeutic option in several types of cancer models (28-30). The growing therapeutic relevance of FGFR alterations, including fusions, in different cancer types, has greatly supported the development of a variety of tyrosine kinase inhibitors (TKIs) (31-33). Although these drugs exhibit good anticancer effects in many, their use in the treatment of brain malignancies is limited. Among the reasons for this is the presence of the blood-brain barrier that influences the delivery of drugs to the central nervous system as well as patient-topatient variability.

The presence of the FGFR3-TACC3 fusion gene certainly represents a further targetable mutation within the molecular heterogeneity typical of the majority of GBMs (34). The reassuring outcome of anti-FGFR inhibitors in different preclinical studies strengthened the rationale to employ FGFR tyrosine kinase inhibitors in GBM patients harboring the FGFR3TACC3 fusion gene $(11,18)$. Different clinical trials studies have been completed (NCT02824133 and NCT01975701) or are still recruiting GBM patients (NCT04424966 or NCT04547855) to test the efficacy of multi-targeted receptor tyrosine kinase inhibitors, such as Anlotinib, or selective FGFR1-3 inhibitors, such as Infigratinib, in relapsed or progressed GBM patients. In this regard, Wang et al. described a partial response ( $>17$ months of follow-up) in a 44-year-old woman affected by recurrent GBM, IDHwt, that harbored simultaneously an FGFR3-TACC3 fusion and FGFR3 amplification, treated with Anlotinib $12 \mathrm{mg}$ p.o. once every day plus oral TMZ chemotherapy (35). Interestingly, the authors speculated that the coexistence of two different FGFR3 alterations (FGFR3-TACC3 fusion and FGFR3 amplification) 
in the same tumor could be the main reason for the significant efficacy of Anlotinib therapy and emphasized that tumors harboring FGFR3-TACC3 rearrangements and/or FGFR3 amplification should be selected for clinical trials featuring FGFR inhibitors (35).

\section{CONCLUSIONS}

The present case highlights that neuropathologists should be aware that the presence of an unusual morphology may reliably predict a distinct molecular profile of GBM, IDHwt, and that, in the presence of the above-mentioned features, they must promptly consider a FGFR3-TACC3 fusion. The spindle cell component embedded in a myxoid stroma, found in our case, contributes to expanding the spectrum of morphologic features that may predict the presence of FGFR3-TACC3 fusions. To this end, the detection of a fusion gene using transcriptome sequencing may represent a novel approach (36). In conclusion, we strongly emphasize that the prompt identification of the combination between unusual morphology and presence of FGFR3-TACC3 fusion has mainly the practical purpose of identifying a subset of patients with a slightly better outcome than those affected by conventional GBM, IDHwt, and for whom the

\section{REFERENCES}

1. Barbagallo D, Caponnetto A, Barbagallo C, Battaglia R, Mirabella F, Brex $\mathrm{D}$, et al. The GAUGAA motif is responsible for the binding between circSMARCA5 and SRSF1 and related downstream effects on glioblastoma multiforme cell migration and angiogenic potential. Int J Mol Sci. (2021) 22:1678. doi: 10.3390/ijms22041678

2. Certo F, Altieri R, Maione M, Schonauer C, Sortino G, Fiumano G, et al. FLAIRectomy in supramarginal resection of glioblastoma correlates with clinical outcome and survival analysis: a prospective, single institution, case series. Oper Neurosurg. (2021) 20:151-63. doi: 10.1093/ons/opaa293

3. Stella M, Falzone L, Caponnetto A, Gattuso G, Barbagallo C, Battaglia R, et al. Serum extracellular vesicle-derived circHIPK3 and circSMARCA5 are two novel diagnostic biomarkers for glioblastoma multiforme. Pharmaceuticals. (2021) 14:618. doi: 10.3390/ph14070618

4. Louis DN, Perry A, Wesseling P, Brat DJ, Cree IA, Figarella-Branger D, et al. The 2021 WHO classification of tumors of the central nervous system: a summary. Neuro Oncol. (2021) 23:1231-51. doi: 10.1093/neuonc/noab106

5. Aldape K, Zadeh G, Mansouri S, Reifenberger G, von Deimling A. Glioblastoma: pathology, molecular mechanisms and markers. Acta Neuropathol. (2015) 129:829-48. doi: 10.1007/s00401-015-1432-1

6. Broggi G, Salvatorelli L, Barbagallo D, Certo F, Altieri R, Tirro E, et al. Diagnostic utility of the immunohistochemical expression of serine and arginine rich splicing factor 1 (SRSF1) in the differential diagnosis of adult gliomas. Cancers. (2021) 13:2086. doi: 10.3390/cancers 13092086

7. Khanna G, Pathak P, Suri V, Sharma MC, Chaturvedi S, Ahuja A, et al. Immunohistochemical and molecular genetic study on epithelioid glioblastoma: Series of seven cases with review of literature. Pathol Res Pract. (2018) 214:679-85. doi: 10.1016/j.prp.2018.03.019

8. Xu T, Wang H, Huang X, Li W, Huang Q, Yan Y, et al. Gene fusion in malignant glioma: an emerging target for next-generation personalized treatment. Transl Oncol. (2018) 11:609-18. doi: 10.1016/j.tranon.2018.02.020

9. Ballester LY, Moghadamtousi SZ, Leeds NE, Huse JT, Fuller GN. Coexisting FGFR3 p.K650T mutation in two FGFR3-TACC3 fusion glioma cases. Acta Neuropathol Commun. (2019) 7:63. doi: 10.1186/s40478-019-0721-7

10. Lasorella A, Sanson M, and Iavarone A. FGFR-TACC gene fusions in human glioma. Neuro Oncol. (2017) 19:475-83. doi: 10.1093/neuonc/now240 use of personalized treatment with FGFR kinase inhibitors may be considered.

\section{AUTHOR CONTRIBUTIONS}

GB, EP, and ET: conceptualization. DC and ET: methodology. $\mathrm{CR}, \mathrm{PV}$, and ET: validation. $\mathrm{GB}$ and ET: formal analysis, writing-original draft preparation, writing-review, and editing. GB: investigation. EP, LC, and CC: resources. RA, FC, and GM: supervision. All authors have read and agreed to the published version of the manuscript.

\section{FUNDING}

This study was partially funded by the Research plan of the University of Catania-Linea di intervento 2-entitled MultiDisciplinary RESEarch and Targeted Therapy for malignant GLIOmas (MD-RESETT-GLIO).

\section{ACKNOWLEDGMENTS}

The authors wish to thank the Scientific Bureau of the University of Catania for language support.

11. Di Stefano AL, Fucci A, Frattini V, Labussiere M, Mokhtari K, Zoppoli $\mathrm{P}$, et al. Detection, characterization, and inhibition of FGFR-TACC fusions in IDH wild-type glioma. Clin Cancer Res. (2015) 21:330717. doi: 10.1158/1078-0432.CCR-14-2199

12. Porta R, Borea R, Coelho A, Khan S, Araujo A, Reclusa P, et al. FGFR a promising druggable target in cancer: molecular biology and new drugs. Crit Rev Oncol Hematol. (2017) 113:256-67. doi: 10.1016/j.critrevonc.2017. 02.018

13. Di Stefano AL, Picca A, Saragoussi E, Bielle F, Ducray F, Villa C, et al. Clinical, molecular, and radiomic profile of gliomas with FGFR3-TACC3 fusions. Neuro Oncol. (2020) 22:1614-24. doi: 10.1093/neuonc/noaa121

14. Bielle F, Di Stefano AL, Meyronet D, Picca A, Villa C, Bernier M, et al. Diffuse gliomas with FGFR3-TACC3 fusion have characteristic histopathological and molecular features. Brain Pathol. (2018) 28:674-83. doi: 10.1111/bpa.12563

15. Gilani A, Davies KD, Kleinschmidt-DeMasters BK. Can adult IDH-wildtype glioblastomas with FGFR3:TACC3 fusions be reliably predicted by histological features? Clin Neuropathol. (2021) 40:165-7. doi: 10.5414/NP301357

16. Mata DA, Benhamida JK, Lin AL, Vanderbilt CM, Yang SR, Villafania LB, et al. Genetic and epigenetic landscape of IDH-wildtype glioblastomas with FGFR3-TACC3 fusions. Acta Neuropathol Commun. (2020) 8:186. doi: 10.1186/s40478-020-01058-6

17. Costa R, Carneiro BA, Taxter T, Tavora FA, Kalyan A, Pai SA, et al. FGFR3TACC3 fusion in solid tumors: mini review. Oncotarget. (2016) 7:5592438. doi: 10.18632/oncotarget.10482

18. Singh D, Chan JM, Zoppoli P, Niola F, Sullivan R, Castano A, et al. Transforming fusions of FGFR and TACC genes in human glioblastoma. Science. (2012) 337:1231-5. doi: 10.1126/science.1220834

19. Broggi G, Certo F, Altieri R, Caltabiano R, Gessi M, Barbagallo GMV. A "polymorphous low-grade neuroepithelial tumor of the young (PLNTY)" diagnosed in an adult. Report of a case and review of the literature. Surg Neurol Int. (2021) 12:470. doi: 10.25259/SNI_500_2021

20. Stupp R, Taillibert S, Kanner AA, Kesari S, Steinberg DM, Toms SA, et al. Maintenance therapy with tumor-treating fields plus temozolomide vs temozolomide alone for glioblastoma: a randomized clinical trial. JAMA. (2015) 314:2535-43. doi: 10.1001/jama.2015.16669

21. Thakkar JP, Dolecek TA, Horbinski C, Ostrom QT, Lightner DD, Barnholtz-Sloan JS, et al. Epidemiologic and molecular prognostic review 
of glioblastoma. Cancer Epidemiol Biomarkers Prev. (2014) 23:198596. doi: 10.1158/1055-9965.EPI-14-0275

22. Taal W, Oosterkamp HM, Walenkamp AM, Dubbink HJ, Beerepoot LV, Hanse MC, et al. Single-agent bevacizumab or lomustine versus a combination of bevacizumab plus lomustine in patients with recurrent glioblastoma (BELOB trial): a randomised controlled phase 2 trial. Lancet Oncol. (2014) 15:94353. doi: 10.1016/S1470-2045(14)70314-6

23. Barthel FP, Johnson KC, Varn FS, Moskalik AD, Tanner G, Kocakavuk E, et al. Longitudinal molecular trajectories of diffuse glioma in adults. Nature. (2019) 576:112-20. doi: 10.1038/s41586-019-1775-1

24. Goenka A, Tiek D, Song X, Huang T, Hu B, Cheng SY. The many facets of therapy resistance and tumor recurrence in glioblastoma. Cells. (2021) 10:484. doi: 10.3390/cells10030484

25. Tirro E, Massimino M, Romano C, Martorana F, Pennisi MS, Stella S, et al. Prognostic and therapeutic roles of the insulin growth factor system in glioblastoma. Front Oncol. (2020) 10:612385. doi: 10.3389/fonc.2020.612385

26. Weller $M$, van den Bent $M$, Preusser $M$, Le Rhun E, Tonn JC, Minniti G, et al. EANO guidelines on the diagnosis and treatment of diffuse gliomas of adulthood. Nat Rev Clin Oncol. (2021) 18:17086. doi: 10.1038/s41571-020-00447-z

27. Pottier C, Fresnais M, Gilon M, Jerusalem G, Longuespee R, Sounni NE. Tyrosine Kinase Inhibitors in cancer: breakthrough and challenges of targeted therapy. Cancers. (2020) 12:731. doi: 10.3390/cancers12030731

28. Stella S, Zammit V, Vitale SR, Pennisi MS, Massimino M, Tirro E, et al. Clinical implications of discordant early molecular responses in CML patients treated with imatinib. Int J Mol Sci. (2019) 20:2226. doi: 10.3390/ijms20092226

29. Tirro E, Massimino M, Stella S, Zammit V, Consoli ML, Pennisi MS, et al. Efficacy of nilotinib in a CML patient expressing the three-way complex variant translocation t(2;9;22). Anticancer Res. (2019) 39:38939. doi: $10.21873 /$ anticanres. 13540

30. Schram AM, Chang MT, Jonsson P, Drilon A. Fusions in solid tumours: diagnostic strategies, targeted therapy, acquired resistance. Nat Rev Clin Oncol. (2017) 14:735-48. doi: 10.1038/nrclinonc.2017.127

31. Stella S, Massimino M, Tirro E, Vitale SR, Scalise L, Leotta S, et al. B-ALL relapses after autologous stem cell transplantation associated with a shift from e1a2 to e14a2 BCR-ABL transcripts: a case report. Anticancer Res. (2019) 39:431-5. doi: 10.21873/anticanres.13130
32. Cohen P, Cross D, Janne PA. Kinase drug discovery 20 years after imatinib: progress and future directions. Nat Rev Drug Discov. (2021) 20:55169. doi: 10.1038/s41573-021-00195-4

33. Medves S, Demoulin JB. Tyrosine kinase gene fusions in cancer: translating mechanisms into targeted therapies. J Cell Mol Med. (2012) 16:23748. doi: 10.1111/j.1582-4934.2011.01415.x

34. Qazi MA, Vora P, Venugopal C, Sidhu SS, Moffat J, Swanton $\mathrm{C}$, et al. Intratumoral heterogeneity: pathways to treatment resistance and relapse in human glioblastoma. Ann Oncol. (2017) 28:1448-56. doi: 10.1093/annonc/mdx169

35. Wang Y, Liang D, Chen J, Chen H, Fan R, Gao Y, et al. Targeted therapy with anlotinib for a patient with an oncogenic FGFR3-TACC3 fusion and recurrent glioblastoma. Oncologist. (2021) 26:173-7. doi: 10.1002/onco.13530

36. Heydt C, Wolwer CB, Velazquez Camacho O, Wagener-Ryczek S, Pappesch $\mathrm{R}$, Siemanowski J, et al. Detection of gene fusions using targeted nextgeneration sequencing: a comparative evaluation. BMC Med Genomics. (2021) 14:62. doi: 10.1186/s12920-021-00909-y

Conflict of Interest: The authors declare that the research was conducted in the absence of any commercial or financial relationships that could be construed as a potential conflict of interest.

Publisher's Note: All claims expressed in this article are solely those of the authors and do not necessarily represent those of their affiliated organizations, or those of the publisher, the editors and the reviewers. Any product that may be evaluated in this article, or claim that may be made by its manufacturer, is not guaranteed or endorsed by the publisher.

Copyright (๑) 2022 Broggi, Piombino, Altieri, Romano, Certo, Barbagallo, Vigneri, Condorelli, Colarossi, Colarossi, Magro and Tirrò. This is an open-access article distributed under the terms of the Creative Commons Attribution License (CC BY). The use, distribution or reproduction in other forums is permitted, provided the original author(s) and the copyright owner(s) are credited and that the original publication in this journal is cited, in accordance with accepted academic practice. No use, distribution or reproduction is permitted which does not comply with these terms. 